\title{
THE EFFECTIVENESS OF SOCIAL MARKETING IN HIV PREVENTION: A LITERATURE REVIEW
}

Janis Duboviks ${ }^{1}$, MSc; Matiss Kite ${ }^{2}$, Pharm.

1,2 University of Latvia, Latvia

\begin{abstract}
Nowadays, social marketing is a widely used approach to address public health issues. Social marketing sells behaviour, making the products, which aims to support the changes in audience behaviour, available to a target audience. One of the most important issues in social marketing today is the evaluation of its effectiveness and its ability to prove that social marketing interventions have led to consistent behavioural changes. Social marketing practitioners are increasingly asked to justify their actions with evidence of effectiveness. Social marketing aims to achieve behavioural changes, thus confirmed changes in health behaviour of the target audience can serve as evidence of its effectiveness. Social marketing is also widely used in HIV prevention, including a variety of behavioural changes and communication.

The aim of the paper is to analyse available evidence on the effectiveness of social marketing in health outcomes and improvements in health behaviour change, with particular emphasis on the effectiveness of social marketing and the methods used to evaluate it in the context of HIV prevention. Adapted form of flow of information through the different phases of a systematic review (PRISMA) was used during the selection process of researches included in this review.

Literature review reveals which social marketing approaches are more often used as a tool to limit the spread of HIV, however it should be noted that most of implemented social marketing campaigns use weak approaches to measure the effectiveness of social marketing, such as measuring health behaviour of the target audience only after implementing a social marketing campaign. In only a few of the studies included in the report, the health behaviour of the target audience and the evaluation of health-related habits were conducted before and after the implementation of the social marketing campaign, which provides stronger evidence of the effectiveness of social marketing.
\end{abstract}

Key words: effectiveness, HIV, social efficiency, social marketing, literature review.

JEL code: M31

\section{Introduction}

In recent decades, Human Immunodeficiency Virus/Acquired Immunodeficiency Syndrome (HIV/AIDS) has become one of the most important health issues worldwide. According to the World Health Organization (WHO), more than 32 million people have died from it so far. In 2018, 770,000 people died of HIV-related causes worldwide. There is no cure for HIV infection. However, individuals can reduce the risk of HIV infection by limiting exposure to risk factors through key approaches to HIV prevention commonly used in combination, such as male and female condoms, HIV screening and counselling, voluntary medical male circumcision, antiretroviral (ART) therapy, and others (World Health Organization, 2019). The experience of the HIV epidemic over the last 20 years shows that there are many ways to prevent HIV. As a result, strategies to control the spread of HIV have been developed and include various forms of behavioural change and communication (Walker D., 2003). A study in Liberia shows that a condom promotion strategy to prevent HIV/Sexually Transmitted Infections (STIs) among highly vulnerable urban youth in a post-conflict environment with limited resources, providing both male and female condoms and information through non-traditional trade places, for instance, music and photo shops, money exchange centres and beauty salons are likely to increase condom use among young people (Harris A. O., Jubwe S., Kennedy S. B., Taylor C. H., Martin R. B., Bee E. M., Perry O. S., Massaquoi M. T., Woods D. V., Barbu E. M., 2011). That study 
demonstrates that traditional marketing approaches and tools are capable of promoting condom use, which is one of the key approaches of the World Health Organization to control the spread of HIV.

In academic literature, the approach that applies traditional marketing principles and techniques to create, communicate and deliver value with the aim of influencing target audience behaviours that benefit society (public health, safety, environment, and communities) as well as target audience is defined as social marketing (Kotler Ph., Lee N. R., 2008). Thus, it can be argued that social marketing aims to benefit society as a whole, or any part thereof, through the behaviour change. Social marketing eases the acceptance, rejection, modification, abandonment or maintenance of certain behaviours (Grier G., Bryant C. A., 2005). This suggests that social marketing selling behaviour, making the products, which aims to support the changes in audience behaviour, available to a target audience.

Nowadays, social marketing is a widely used approach to addressing public health issues including tobacco use, obesity, teenage pregnancy, HIV/AIDS and high cholesterol (Cheng H., Kotler Ph., Lee N., 2011). However, despite the widespread use of social marketing world increasingly rise many questions of its effectiveness. For instance, Andreasen argues that awareness raising on an issue among target audience does not always provide convincing evidence that the goals of social marketing interventions have been achieved, but it may support the end goal of social marketing (Andreasen A. R., 2002). It seems that in today's economic rationalism the issue of evidence of effectiveness has become more relevant. Therefore, one of the most important issues in social marketing today is the evaluation of its effectiveness and its ability to prove that social marketing interventions have led to consistent behavioural changes. It means, that social marketing practitioners need to identify relevant determinants of health, choose activities to promote health, and then evaluate the effectiveness of these chosen activities (Raphael D., 2000). As it follows from the definition of social marketing, it aims to achieve behavioural changes, thus it can be concluded that confirmed changes in health behaviour of the target audience can serve as evidence of its effectiveness (Firestone R., Rowe C. J., Modi Sh. N., Sievers D., 2017). While the ability of social marketing practitioners to identify relevant health determinants and to choose appropriate health promotion activities is not currently disputed, there is still debate about the evidence of the effectiveness of social marketing.

The aim of the paper is to analyse available evidence on the effectiveness of social marketing in health outcomes and improvements in health behaviour change, with particular emphasis on the effectiveness of social marketing and the methods used to evaluate it in the context of HIV prevention. In order to reach the aim of the study, the following tasks were indicated: 1) identify and review previously done systematic reviews that touch on the effectiveness of social marketing for global health; 2) set and use appropriate methodology in research selection process and data collection and summarizing; 3 ) select and analyse available scientifically based evidence on the effectiveness of social marketing in health outcomes and improvements in health behaviour change in the context of HIV prevention; 4) analyse and interpret gained data in order to identify methods and indicators used to evaluate social marketing efficacy in the context of HIV prevention; 5) summarize the results of the research to draw conclusions. The object of the study is available evidence on the effectiveness of social marketing in health outcomes and improvements in health behaviour change in the context of HIV prevention, and the subject of the study is the methods and indicators used to evaluate the effectiveness of social marketing in the context of HIV prevention. Qualitative and quantitative content analysis were used, also the adopted form of flow of information 
through the different phases of a systematic review (PRISMA) (Moher D., Liberati A., Tetzlaff J., Altman D. G., 2009) was applied during paper selection.

Given the growing role of social marketing in global health in recent decades, researchers have previously conducted studies to determine and reflect its effectiveness, based on evaluations of past social marketing campaigns.

\section{Existing reviews on the effectiveness of social marketing}

A systematic review of the literature is one of the most popular methods in social research to identify available research evidence on a particular subject. The goal is transparent and rigorous review of the evidence available to improve the opinion of the validity and reliability of the findings (Truong V. D., Dang Vu N. V. H., 2016). Several systematic reviews on the effectiveness of social marketing in global health were identified, covering topics such as HIV prevention, drug use and child morality. Some researchers who have previously conducted a literature review on the effectiveness of social marketing indicate that one of the most important challenges of systematic literature reviews is defining social marketing interventions, because there is a risk that social marketing is misclassified as health promotion or social and behaviour change communications (Firestone R., Rowe C. J., Modi Sh. N., Sievers D., 2017; Sewak A., Singh G., 2017; Sipe T. A., Barham T. L., Johnson W., Joseph H., Tungol-Ashmon M. L., O'Leary A., 2017; Truong V. D., Dang Vu N. V. H., 2016). For example, some practitioners define social marketing as the use of strategic marketing to promote social change (Robinson M. N., Tansil K.A., Elder R. W., Soler R. E., Labre M. P., Mercer S. L., Eroglu D., Baur C., Lyon-Daniel K., Fridinger F., Sokler L. A., Green L. W., Miller T., Dearing J., Evans W. D., Snyder L. B., Viswanath K. K., Beistle D. M., Chervin D. D., Bernhardt J. M., Rimer B. K., 2014), some, instead if social marketing definition, use definition such as mass communication campaigns focused on sexual behaviour, HIV testing or both (Noar S. M., Palmgreen Ph., Chabot M., Dobransky N., Zimmerman R. S., 2009). As well in previously done reviews can be found definitions such as health promotion intervention, in particular behaviour change communication strategies, which utilize different communication channels to promote positive health behaviours at individual and community levels (Juma K., Reid M., Roy M., Vorkoper S., Temu T. M., Levitt N. S., Oladepo O., Zakus D., Yonga G., 2018) or condom social marketing (Sweat M. D., Denison J., Kennedy C., Tedrow V., O'Reilly K., 2012). Before conducting a systematic review of the literature, it is important to define the exact criteria by which the papers will be selected. However, in some previous studies, practitioners did not define social marketing at all or did not define health promotion or social and behavioral change as inclusion criteria in the review (Luca N. R., Suggs L. S., 2010; Stromdahl S., Hickson F., Pharris A., Sabido M., Baral S., Thorson A., 2015; Werb D., Buxton J., Shoveller J., Richardson C., Rowell G., Wood E., 2013; McDaid L., Riddell J., Teal G. Boydell N., Coia N., Flowers P., 2019). In a situation where researchers are differently interpreting and defining social marketing and its tasks, it is difficult to track the evidence of its effectiveness, but this is of major interest to the global health community today (Firestone R., Rowe C. J., Modi Sh. N., Sievers D., 2017). Thereby, when conducting a literature review, it is important to clearly and unambiguously define not only paper inclusion criteria but also social marketing, thus ensuring that evidence of its effectiveness is available to global health community and other interested parties.

Likewise, the previous literature reviews on the effectiveness of social marketing do not fully reflect the measurement methods and factors used, making it difficult to determine a complete set 
of methods and factors for assessing the effectiveness of social marketing and providing reliable evidence of its effectiveness.

\section{Methodology}

Social marketing campaigns was defined as campaigns using traditional marketing principles and techniques to influence target audience behaviours to improve public's health and change its health behaviour. Studies were included if: (1) behaviour-change is the benchmark used to design and evaluate interventions; (2) intervention focuses on the audience, fully understands their lives, behaviour and the issue using a mix of data sources and research methods; (3) the central element of any influence strategy is creating attractive and motivational exchanges with target audiences; (4) careful attention is paid to the competition faced by the desired behaviour; (5) studies were original research and included original data, evaluation method(s) were reported; (6) published in English; (7) assessed a campaign that attempted to change a behavioural factor, health outcome or behaviour within area of HIV prevention. All editorial or review studies were excluded. Also economic evaluations were excluded, except those studies were was possible to identify behaviour factor, behaviour or health outcome results.

Authors searched for studies published from 1983 to 2018 that evaluated the effectiveness of social marketing programmes in the HIV area using the bibliographic database Scopus. The search period of published articles was based on the fact that the first clinical evidence of AIDS was reported in June 1981 and its cause, HIV, was identified in 1983 (United Nations, 2019). Titles, abstracts and keywords were searched for the following terms: ("social marketing" OR "social marketing theory" OR "social franchise" OR "social franchises" OR "social franchising") AND ("HIV" OR "HIV prevention" OR "AIDS"). After duplicates were removed, authors reviewed titles and abstracts if they met eligibility criteria. The selection process and data extraction were compared between two authors and, if there was concern about article inclusion, the abstract was reviewed repeatedly, and inconsistencies were addressed through discussion. Full texts of studies were reviewed by both authors, and all inconsistencies also were addressed through discussion.

\section{Research results and discussion}

The initial search identified 502 records using the described search terms. Of those, 43 duplicates and reviews were removed. Then authors reviewed 459 articles at the abstract level. In result, 330 studies were excluded based on the above inclusion criteria. Full text review resulted with another 76 articles elimination, yielding 53 eligible studies for inclusion in analysis. The selection process is described in Figure 1 using adapted form of flow of information through the different phases of a systematic review.

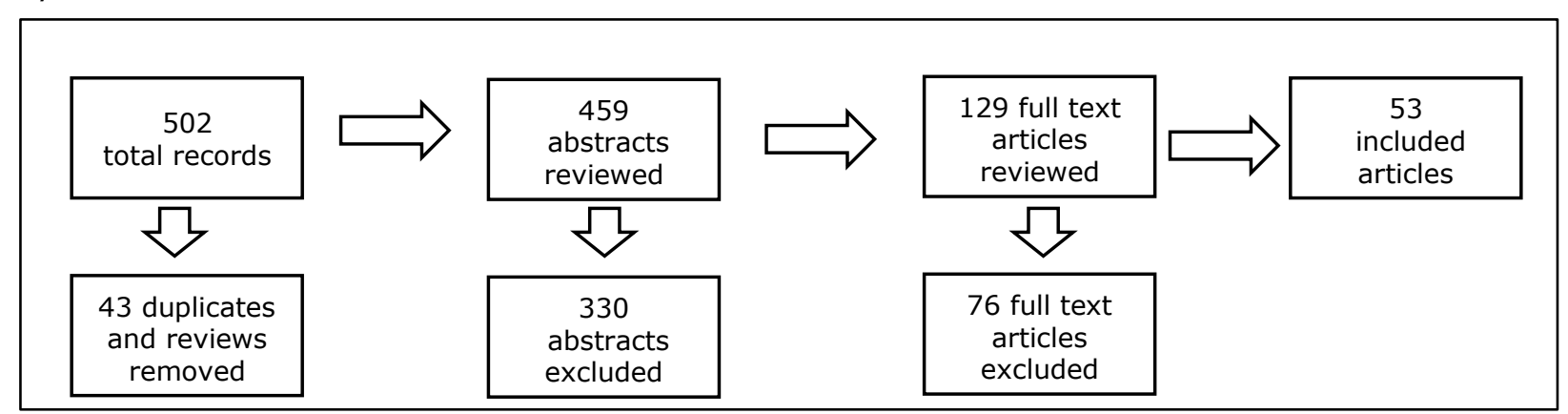

Source: author's construction based on data collection results (June, 2019)

Fig. 1. Flow diagram 
Data in Table 1 represent that the greatest number of studies focused on HIV testing and condom use promotion, followed by educational communications, ART promotion, reduce of needle sharing and mix campaign focused on both reduce of needle sharing and condom use promotion. This distribution of marketing campaign focus data shows that most of the campaigns implemented comply with WHO' s HIV prevention recommendations.

Table 1

Included studies by campaign focus

\begin{tabular}{|l|l|c|c|}
\hline No & \multicolumn{1}{|c|}{ Campaign focus } & Number & $\%$ \\
\hline 1. & HIV testing & 19 & $35.85 \%$ \\
\hline 2. & Condom use & 19 & $35.85 \%$ \\
\hline 3. & Educational communication & 12 & $22.63 \%$ \\
\hline 4. & ART promotion & 1 & $1.89 \%$ \\
\hline 5. & Reduce of needle sharing & 1 & $1.89 \%$ \\
\hline 6. & Reduce of needle sharing/ condom use & 1 & $1.89 \%$ \\
\hline & & 53 & $100 \%$ \\
\hline
\end{tabular}

Source: author's construction based on data collection results (June, 2019)

In 41 of the papers included in this review, researchers evaluated the effectiveness of social marketing based on data of changes in health outcomes or health behaviour of the campaign's target audience gathered after social marketing intervention implementation only. In turn, 12 studies evaluated the effectiveness of a social marketing intervention based on the data of health outcomes and health behaviour of the target audience obtained pre- and post the intervention. In Table 2 is summarized data on included studies by social marketing campaign focus and applied method of social marketing effectiveness evaluation.

Table 2

Included studies by applied method of social marketing effectiveness evaluation

\begin{tabular}{|l|l|c|c|c|c|}
\hline No & \multicolumn{1}{|c|}{ Campaign focus } & $\begin{array}{c}\text { Comparative } \\
\text { statistics }\end{array}$ & Survey & $\begin{array}{c}\text { Comparative } \\
\text { statistics/ } \\
\text { focus group } \\
\text { interview }\end{array}$ & Interview \\
\hline 1. & HIV testing & 13 & 4 & 0 & 2 \\
\hline 2. & Condom use & 7 & 12 & 0 & 0 \\
\hline 3. & Educational communication & 5 & 6 & 1 & 1 \\
\hline 4. & ART promotion & 1 & 0 & 0 & 0 \\
\hline 5. & Reduce of needle sharing & 1 & 0 & 0 & 0 \\
\hline 6. & Reduce of needle sharing/ condom use & 1 & 0 & 0 & 0 \\
\hline
\end{tabular}

Source: author's construction based on data collection results (June, 2019)

As Table 2 reflects, the most commonly used methods are comparative statistics and survey. But qualitative research methods such as focus group interviews and in-depth interviews were used in only 4 of the studies included in this review. Indicators such as the number of performed HIV tests comparing to a similar period before the intervention were evaluated using comparative statistical methods, when the evaluation of the effectiveness of intervention was performed only after intervention implementation. Studies that collected data on the health behaviour of the target 
audience before and after the intervention compared the number of HIV tests performed in the target audience, rather than general statistics.

In evaluation of the effectiveness of intervention focused on condom use promotion comparative statistics was also used. But it should be noted, that given the sensitivity of the topic and the near impossibility of the researcher's ability to check the use of condom in order to determine whether health behavioural changes have occurred, in this case the assessment of effectiveness of intervention was done by comparing the sales of condoms before and after the intervention, or comparing of the rise/fall of condom purchases within the relevant target audience. In papers included in this review such indicators as unique page views/visits, ad audience reach rates, etc. within the target audience before or after the intervention were used to evaluate its effectiveness. Popularisation of ART among already infected patients included measures of an increase in the number of patients who started ART or resumed ART after a long interruption. To evaluate the effectiveness of the marketing intervention focused on the syringe exchange campaigns, data on new dispensed syringes as well as the decrease/increase in newly discovered HIV cases among intravenous drug users who were the target audience of this intervention were used.

The second most popular method of evaluating the effectiveness of social marketing intervention is to survey the audience after pre and post intervention implementation. Depending on focus of an intervention the questions asked were: how often the respondents did HIV test; how often they used condom, whether they reduced the number of sexual partners, what their knowledge about HIV was at that moment etc. Authors believe, the survey is a good method for measuring a social marketing intervention that focuses on raising awareness, as the survey can show quite accurately how current knowledge on HIV issues has changed. Moreover, to see a clear trend in knowledge change is an imperative to conduct a pre and post campaign survey. However, the survey is not able to fully reflect the behavioural changes of the target audience, as respondents are often more inclined to report better results than they really are. For example, in authors' opinion, the survey as evaluation tool of effectiveness of intervention focused on HIV testing and condom use promotion does not fully reflect the reality, but rather indicates respondents' intention to act "right" in order to protect themselves from HIV infection.

Analysing in this review included researches, it should be emphasized that better results in health outcomes and behavioural changes of the target audience were reported in campaigns that provided access to behavioural products alongside educational communication (e.g. condom provision, specified places where it was possible to take free HIV test, immediate availability of syringes etc.), thus providing a better exposition of behavioural product for target audience.

Any marketing campaign must have a well-defined target audience in order to measure its effectiveness, otherwise it is difficult to evaluate its effectiveness, otherwise, it rises doubts that the campaign's creators have been aware of its needs and current situation, including cultural, social, demographic and other factors.

When evaluating the effectiveness of a social marketing campaign, it is important to understand its long-term rather than short-term, impact on the health outcome and health behaviour of the target audience, which is the most important purpose and role of social marketing. Evaluating the effectiveness of a marketing campaign immediately after its implementation weakens the evidence of the effectiveness of social marketing as a tool which is able to improve public health and change health behaviour, because inconsistent changes in the health outcomes or health behaviour of the target audience in the direction that campaign's creators want to achieve are not scientifically proven. 
Based on the results of the review, it could be concluded that in order to create effective social marketing intervention, it is necessary to carefully explore the target audience with a full understanding of their lifestyle, needs and other factors affecting their lives and knowledge. In order to increase the effectiveness of social marketing intervention, it is necessary to evaluate the possibility of providing the best exposition of behavioural products to target audience. To determine the real effectiveness of social marketing interventions, several factors need to be taken into account, and increased attention needs to be paid to assessing the long-term effectiveness of a social marketing intervention, as in the literature there is not much evidence available for it.

\section{Limitations}

This review has several limitations. Only studies in which the evaluation of effectiveness of social marketing interventions could be identified were included in this review. In addition, given the variety of variations in the definition of social marketing in academic literature, there is possibility that authors unintentionally included in the review a study that is essentially an evaluation of a health promotion campaign rather than an evaluation of an implemented social marketing intervention. Likewise, there is another possibility that social marketing intervention has been misclassified due to the minimal availability of information in published articles.

\section{Conclusions, proposals, recommendations}

1) Social marketing is widely used in the prevention of the spread of HIV by application of traditional marketing tools and approaches for its implementation including a variety of health behaviour changes and forms of communication.

2) Most social marketing campaigns focus on reducing the impact of health risk factors determined by World Health Organization.

3) Providing a product that can potentially influence changes in health behaviour of target audience during the campaign available to particular audience increases campaign's effectiveness and promotes behaviour change more effectively comparing to interventions when product is not available to target audience.

4) Most popular methods of evaluation of effectiveness of social marketing campaigns devoted to HIV prevention are comparative statistics and target audience survey.

5) Majority of social campaigns included in the review run poor approaches to measuring the effectiveness of social marketing interventions, such as measuring changes in health outcomes and health behaviours of the target audience only after its implementation, which weakens the evidence of its effectiveness, and ignore various factors such as product availability to target audience, cultural factors, and target audience knowledge on the issue.

6) Measuring the effectiveness of a campaign immediately after its implementation weakens evidence of its effectiveness because it does not demonstrate consistent long-term changes in health-related habits, which is the main purpose of social marketing.

7) Using the audience survey as a method to measure the effectiveness of the campaign can weaken the evidence of its effectiveness because respondents are not always willing to indicate the true action, but only the intention. However, that given the sensitivity of the topic, such as changes in condom use or sexual lifestyle, it should be acknowledged that survey remains almost the only method of evaluation. 
8) Campaign's creators are recommended to take into account that in order to create an effective social marketing intervention, it is necessary to carefully explore the target audience and provide the best possible exposition of behavioural products to target audience during the campaign.

9) In the future, the increased attention needs to be paid to evaluation of the effectiveness of social marketing intervention in a long-term as there is a lack of evidence for it in literature.

\section{Bibliography}

1. Andreasen, A. R. (2002). Marketing Social Marketing in the Social Change Marketplace. Journal of Public Policy and Marketing, Volume 21, Issue 1, pp. 3 - 13.

2. Cheng, H., Kotler, Ph., Lee, N. (2011). Social Marketing for Public Health: Global Trends and Success Stories. Sudbury, Mass.: Jones and Bartlett, p. xxv, 442.

3. Firestone, R., Rowe, C. J., Modi, Sh. N., Sievers, D. (2017). The Effectiveness of Social Marketing in Global Health: A Systematic Review. Health Policy and Planning, no 32, pp. $110-124$.

4. Grier, G., Bryant, C. A. (2005). Social Marketing in Public Health. Public Health, no 39, pp. 319 - 339.

5. Harris, A. O., Jubwe, S., Kennedy, S. B., Taylor, C. H., Martin, R. B., Bee, E. M., Perry, O. S., Massaquoi, M. T., Woods, D.V., Barbu, E. M. (2011). Condom Social Marketing Program to Prevent HIV/AIDS in Post-Conflict Liberia. African Health Sciences, no 11, pp. S77 - S81.

6. Juma, K., Reid, M., Roy, M., Vorkoper, S., Temu, T. M., Levitt, N. S., Oladepo, O., Zakus, D., Yonga, G. (2018). From HIV Prevention to Non-Communicable Disease Health Promotion Efforts in Sub-Saharan Africa. AIDS, no 32, pp. S63 - S73.

7. Kotler, Ph., Lee, N. R. (2008). Social Marketing: Influencing Behaviors for Good (3rd ed.). Los Angeles; London; New Delhi; Singapore: Sage Publications, p. xii, 444.

8. Luca, N. R., Suggs, L. S. (2010). Strategies for the Social Marketing Mix: A Systematic Review. Social Marketing Quarterly, Volume 16, Issue, 4, pp. 122 - 149.

9. McDaid, L., Riddell, J., Teal, G. Boydell, N., Coia, N., Flowers, P. (2019). The Effectiveness of Social Marketing Interventions to Improve HIV Testing Among Gay, Bisexual and Other Men Who Have Sex with Men: A Systematic Review. AIDS Behaviour, Volume 23, Issue 9, pp. $2273-2303$.

10. Moher, D., Liberati, A., Tetzlaff, J., Altman, D. G. (2009). Preferred Reporting Items for Systematic Reviews and Meta-Analyses: The PRISMA Statement. BMJ - British Medical Journal, no 339, pp. 332 - 336.

11. Noar, S. M., Palmgreen, Ph., Chabot, M., Dobransky, N., Zimmerman, R. S. (2009). A 10-Year Systematic Review of HIV/AIDS Mass Communication Campaigns: Have We Made Progress? Journal of Health Communication, no 14, pp. $15-42$.

12. Raphael, D. (2000). The Question of Evidence in Health Promotion. Health Promotion International, Volume 15, Issue 4, pp. $355-367$

13. Robinson, M. N., Tansil, K.A., Elder, R. W., Soler, R. E., Labre, M. P., Mercer, S. L., Eroglu, D., Baur, C., LyonDaniel, K., Fridinger, F., Sokler, L. A., Green, L. W., Miller, T., Dearing, J., Evans, W. D., Snyder, L. B., Viswanath, K. K., Beistle, D. M., Chervin, D. D., Bernhardt, J. M., Rimer, B. K. (2014). Mass Media Health Communication Campaigns Combined with Health-Related Product Distribution: A Community Guide Systematic Review. American Journal of Preventive Medicine, Volume 47, Isuue 3, pp. 360 - 371.

14. Sewak, A., Singh, G. (2017). Integrating Social Marketing into Fijian HIV/AIDS Prevention Programs: Lessons from Systematic Review. Health Communications, Volume 32, Issue 1, pp. $32-40$.

15. Sipe, T. A., Barham, T. L., Johnson, W., Joseph, H., Tungol-Ashmon, M. L., O'Leary, A. (2017). Structural Interventions in HIV Prevention: A Taxonomy and Descriptive Systematic Review. AIDS Behaviour, Volume 21 , Issue 12, pp. $3366-3430$.

16. Stromdahl, S., Hickson, F., Pharris, A., Sabido, M., Baral, S., Thorson, A. (2015). A Systematic Review of Evidence to Inform HIV Prevention Interventions among Men Who Have Sexith Men in Europe. Euro Surveill 20 (15). Retrieved: http://www.eurosurveillance.org/ViewArticle.aspx?ArticleId=21096 Access: 27.08.2019.

17. Sweat, M. D., Denison, J., Kennedy, C., Tedrow, V., O'Reilly, K. (2012). Effects of Condom Social Marketing on Condom Use in Developing Countries: A Systematic Review and Meta-Analysis, 1990-2010. Bull World Health Organization, Volume 90, Issue 8, pp. 613 - 622A.

18. Truong, V. D., Dang Vu, N. V. H. (2016). Reviewing Research Evidence for Social Marketing: Systematic Literature Reviews in: Kubacki, K., Rundle-Thiele, S. (2016). Formative Research in Social Marketing, pp. 183 $-250$.

19. United Nations. AIDS. (2019). Retrieved: http://www.un.org/en/sections/issues-depth/aids/index.html. Access: 29.03.2019.

20. Walker, D. (2003). Cost and Cost-Effectiveness of HIV/AIDS Prevention Strategies in Developing Countries: Is There an Evidence Base? Health Policy and Planning, Volume 18, Issue 1, pp. $4-17$.

21. Werb, D., Buxton, J., Shoveller, J., Richardson, C., Rowell, G., Wood, E. (2013). Interventions to Prevent the Initiation of Injection Drug Use: A Systematic Review. Drug and Alcohol Dependence, no 133, pp. 669 - 676.

22. World Health Organization. HIV/AIDS: Key Facts. (2019). Retrieved: https://www.who.int/news-room/factsheets/detail/hiv-aids Access: 26.08.2019. 